\title{
BILATERAL SENSORINEURAL HEARING LOSS FOLLOWING NON OTOLOGIC SURGERY
}

Poonam K. Saidha ${ }^{1}$, Sreenivas V²

\section{HOW TO CITE THIS ARTICLE:}

Poonam K. Saidha, Sreenivas V. "Bilateral Sensor neural Hearing Loss following Non Otologic Surgery". Journal of Evolution of Medical and Dental Sciences 2014; Vol. 3, Issue 43, September 11; Page: 10697-10703,

DOI: $10.14260 /$ jemds/2014/3394

INTRODUCTION: A 58 years old man with a no history of hearing loss was admitted with comminuted fracture tibia and fibula (right), sustained in a road traffic accident [Fig. 1]. He had no known comorbidity. The pre-operative history and physical examination, blood test investigations, chest radiograph [Fig. 2] and ECG were unremarkable.

He was taken up for open reduction and pinning of fractured segments [Fig. 3]. The orthopedic surgery itself was uneventful with the patient being under general anesthesia for a total of one hour. Induction of anesthesia was uncomplicated, and blood loss was minimal. The blood pressure and pulse remained stable, with minimum diastolic and systolic pressure during surgery being $70 \mathrm{mmHg}$ and $130 \mathrm{mmHg}$ respectively. Oxygen saturation remained over 95 percent.

In the post-operative ward, he received parenteral antibiotic cover with Inj Augmentin. Additionally he was given Inj Diclofenac for analgesia and Inj Ondensatron for nausea. Shortly after transfer to the ward, he complained of tinnitus and being unable to hear in either ear. There was no nausea, vomiting, or vertigo.

An otolaryngology consultation at this time showed normal cranial nerve, cerebellar, oculomotor and fundoscopic exams. Audiometry showed bilateral profound $100 \mathrm{~dB}$ and $105 \mathrm{~dB}$ sensorineural hearing loss in the right and left ears respectively [Fig. 4]. Blood tests including sedimentation rate, coagulation profile, blood count, smear examination, lipid profile, serum thyroid hormone levels, renal and liver function tests were all normal.

CT scan of skull was normal except for mild diffuse cerebral atrophy [Fig. 5, 6, 7].

He was placed on conservative management with oral steroids (50 mg/day) for 10 days.

Serial audiograms of the patient thereafter showed mild improvement, with residual hearing loss in both ears of $80 \mathrm{~dB}$. After four weeks, there continues to be hearing loss in both ears [Fig. 8]. Speech comprehension continues to be difficult for the patient, with speech discrimination scores being less than 50 percent in both ears.

DISCUSSION: The impact of non-otologic surgery associated hearing loss on the patient can be distressing, particularly if the hearing loss is bilateral. Only few cases of the bilateral non-otologic surgery associated hearing loss have been described in literature. Hocherman and Reimer describe a case in which the hearing loss recovered substantially in one ear.[6]

In our case there has been severe permanent bilateral hearing loss. The severity is more marked than the pure tone threshold may indicate, because the speech discrimination score remains less than 50 percent.

The mechanism by which sudden sensorineural hearing loss occurs, either sporadically or post operatively is unknown. Some theories are speculative at best. 
Micro embolisms generated in the pump oxygenator systems with occlusion of the cochlear branch of the internal auditory artery are considered the most likely cause of sensorineural hearing loss following cardiovascular surgery.[7]

Micro embolisms in the inner ear can cause ischemia of the striavascularis and hair cells, which may result in irreversible hearing impairment. ${ }^{[8]}$

Hemodynamic fluctuations such as prolonged perioperative hypotension or perfusion failure during cardio-pulmonary by-pass have been proposed as a cause of sensorineural hearing loss following cardiac surgery.[9] In our patient, his vital parameters were stable throughout the operation, and there was no period of hypotension.

Drugs frequently associated with ototoxicity include aminoglycosides, erythromycin, vancomycin, loop diuretics, salicylates, and non-steroidal anti-inflammatory agents.[8] Our patient, however, did not receive any potentially ototoxic drugs in concentrations that could cause ototoxicity.

Hypercoagulable states potentiating thromboembolism may also cause sensorineural hearing loss. Our patient did not receive blood products or drugs such as tranexamic acid that can result in a hypercoagulable state. Laboratory tests showed that his coagulation state was normal.

Sensorineural hearing loss may also be caused by injury to the central nervous system, but as our patient did not show any other abnormal neurological findings, and the CT scan was normal, this was very unlikely in our patient.

The mainstay of treatment, if any, is usually based on corticosteroid administration regardless of the initiating cause of sensorineural hearing loss. Patients with hearing impairment after cardio-pulmonary bypass have been shown to have a poorer prognosis than patients who experience hearing loss after other procedures, with less than $50 \%$ of the former showing recovery.[10] The initial shape of the audiogram has been found to have significant correlation with the extent of recovery.[11]

Hearing impairment in the high frequency range is considered an unfavorable factor, whereas a low to middle frequency audiogram contour is associated with an increased likelihood of recovery. The hearing loss in our patient was confirmed by audiometry to be mainly in the high frequency range. He showed poor signs of recovery despite steroid treatment.

In conclusion, SNHL may occur after various procedures, and those following surgeries using $\mathrm{CPB}$ are more common. Although there have been many case reports related to SNHL after CBP, the exact incidence of hearing loss after non-otologic surgery is unknown and, in view of very limited reports in literature, presumed to be extremely rare.

\section{REFERENCES:}

1. By l FM Jr. Sudden hearing loss: eight years' experience and suggested prognostic table. Laryngoscope. 1984; 94: 647-661.

2. Ashraf 0 . Coronary artery by-pass grafting and sensorineural hearing loss, a cohort study. BMC Ear Nose Throat Disorders. 2005; 5: 12.

3. Shapiro MJ, Purn JM, Raskin C. A study of the effects of cardiopulmonary bypass surgery on auditory function. Laryngoscope. 1981; 91: 2046-2052.

4. De la Cruz M, Bance M. Bilateral sudden sensorineural hearing loss following non-otologic surgery. J Laryngol Otol. 1998; 112: 769-771.

5. Jaffe BF. Suden deafness-an otologic emergency. Archives of Otolaryngology 1967; 86: 55-60. 


\section{CASE REPORT}

6. Hochermann M, Reimer A. hearing loss after general anesthesia (A case report and review of literature). J Laryngol \& Otol. 1987; 101: 1079-82.

7. Walsted A, Andreassen UK, Berthelsen PG, Olesen A. Hearing loss after cardiopulmonary bypass surgery. Eur Arch Otorhinolaryngol. 2000; 257: 124-127.

8. Sprung J, Bourke DL, Contreras MG, Warner ME, Findlay J. Perioperative hearing impairment. Anesthesiology. 2003; 98: 241-257.

9. Shapiro MJ, Purn JM, Raskin C. A study of the effects of cardiopulmonary bypass surgery on auditory function. Laryngoscope. 1981; 91: 2046-2052.

10. Temporary bilateral sensorineural hearing loss following cardiopulmonary bypass - A case report. Korean J Anesthesiol. 2011 August; 61(2): 162-165.

11. Xenellis J, Karapatsas I, Papadimitriou N, Nikolopoulos T, Maragoudakis P, Tzagkaroulakis M, et al. Idiopathic sudden sensorineural hearing loss: prognostic factors. J LaryngolOtol 2006; 120: 718-724.

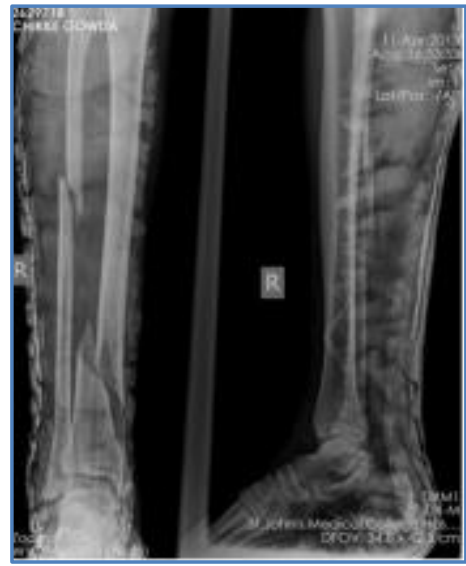

Fig. 1: Communited fracture tibia and fibula

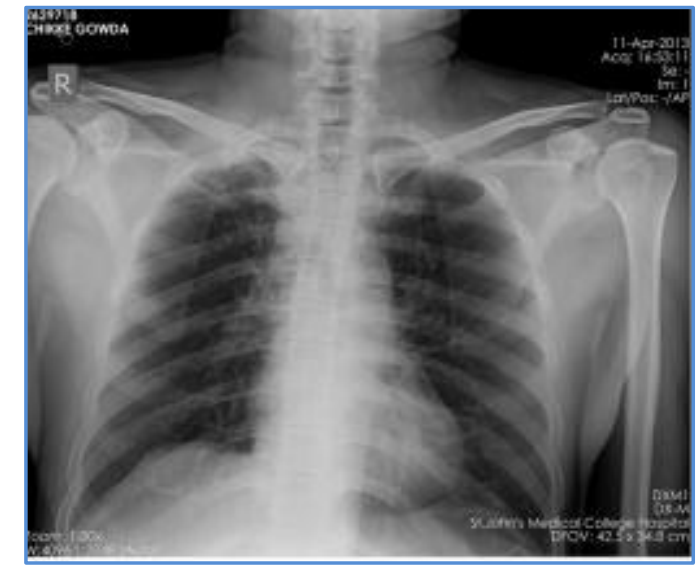

Fig. 2: Preoperative chest radiograph

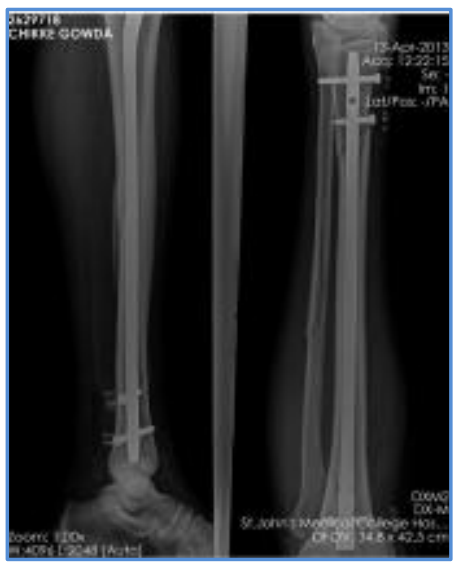

Fig. 3: Open reduction and internal fixation of fracture segments 


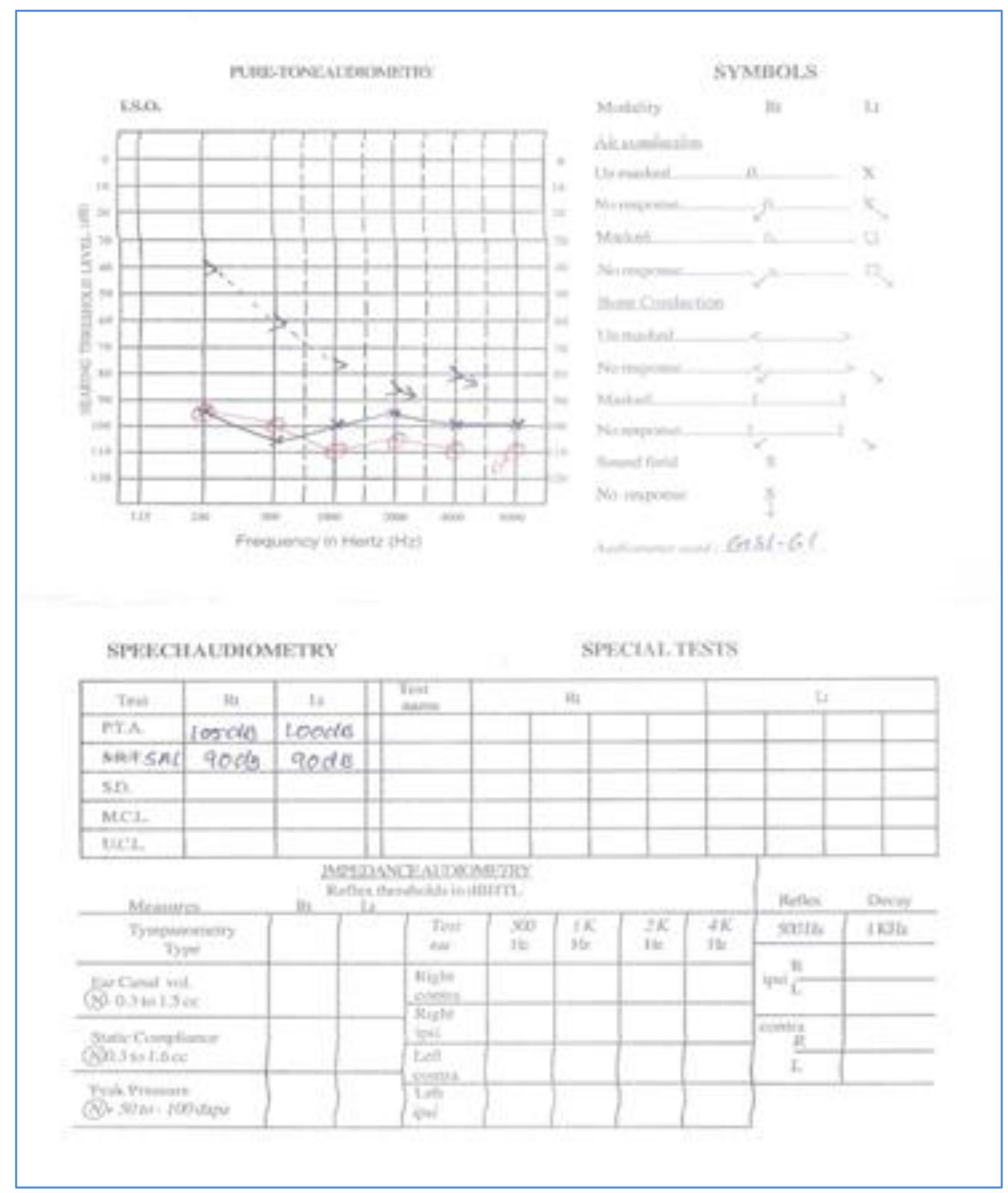

Fig. 4: Post op audiometry showing hearing loss 


\section{CASE REPORT}

Figure 5, 6 and 7 CT Brain showing no abnormality

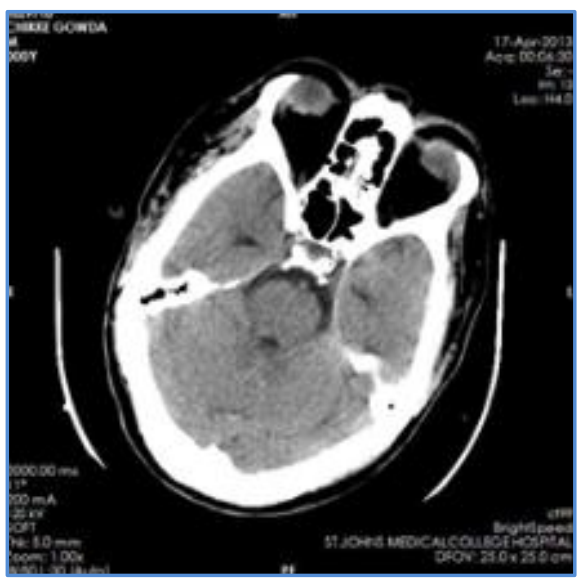

Fig. 5

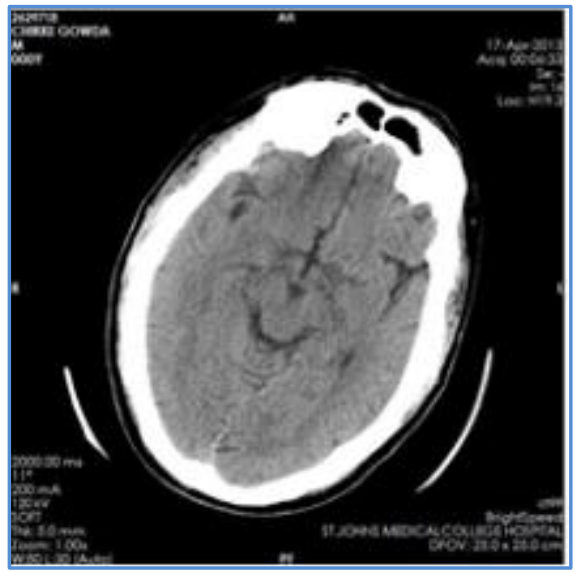

Fig. 6

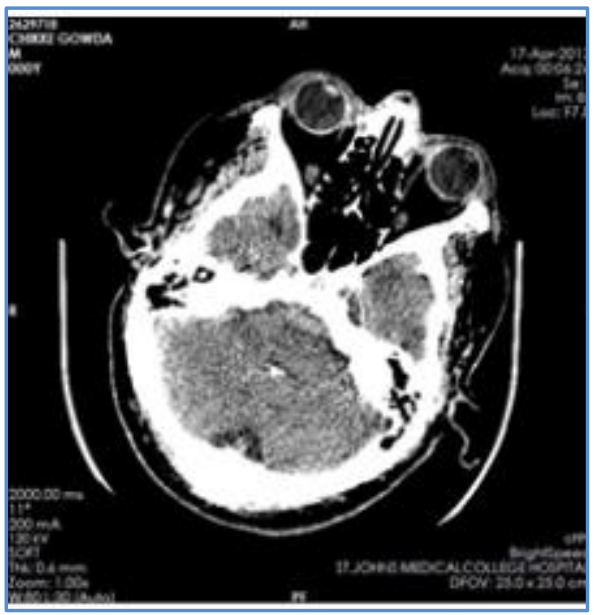

Fig. 7 


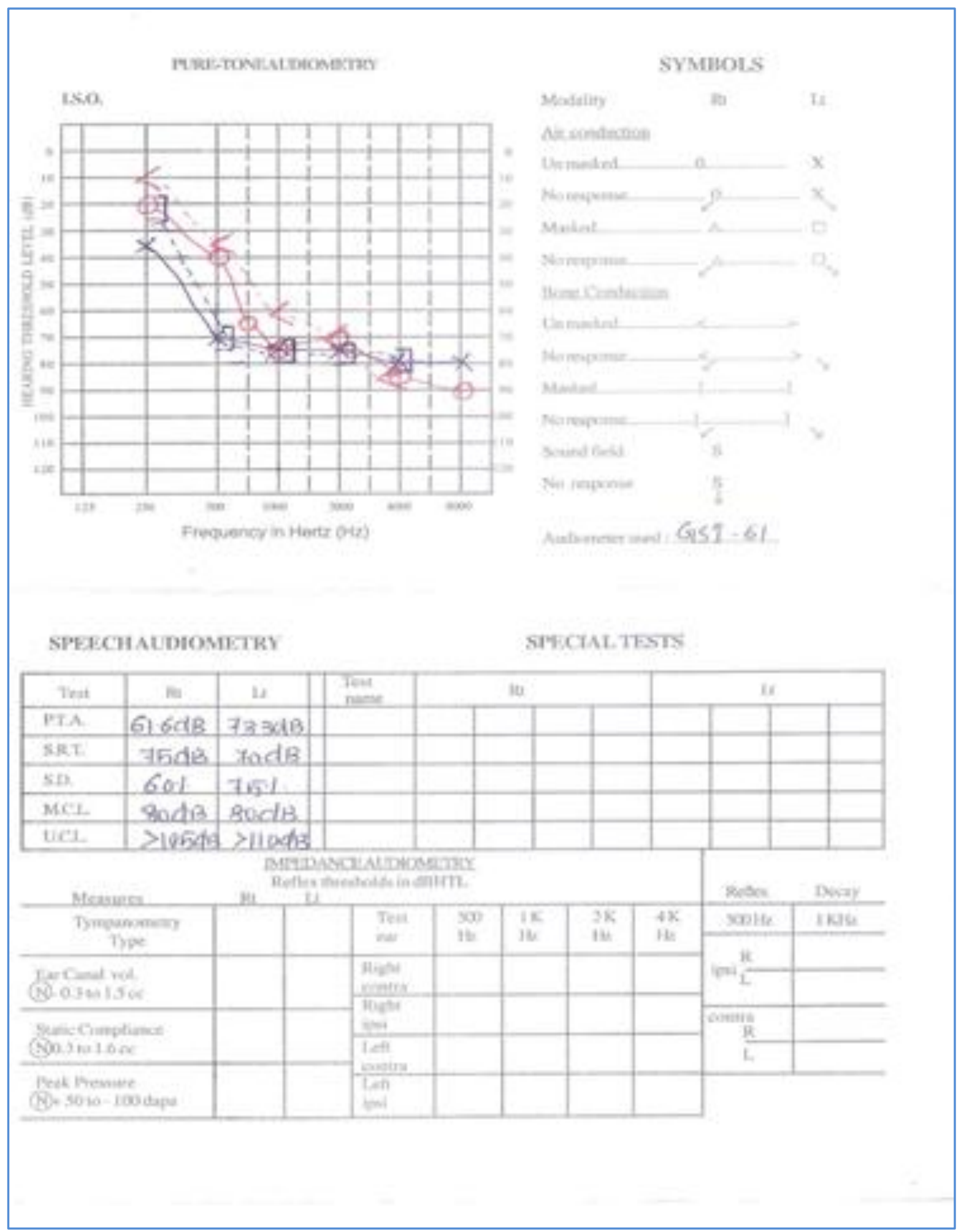

Fig. 8: Residual hearing loss 4 weeks post-surgery 


\section{CASE REPORT}

\section{AUTHORS:}

1. Poonam K. Saidha

2. Sreenivas V.

\section{PARTICULARS OF CONTRIBUTORS:}

1. ENT Specialist, Department of ENT, St. John's Medical College and Hospital, Bangalore.

2. Associate Professor, Department of ENT, St. John's Medical College and Hospital, Bangalore.

\section{NAME ADDRESS EMAIL ID OF THE}

\section{CORRESPONDING AUTHOR:}

Poonam K. Saidha,

Department of ENT,

St. John's Medical College and Hospital,

Bangalore.

Email: nksaidha@gmail.com

Date of Submission: 15/08/2014.

Date of Peer Review: 16/08/2014.

Date of Acceptance: 06/09/2014.

Date of Publishing: 10/09/2014. 